ARTICLE

\title{
Chemical synthesis of a two-photon-activatable chemokine and photon-guided lymphocyte migration in vivo
}

Xin Chen ${ }^{1, \star}$, Shan Tang ${ }^{2, \star}$, Ji-Shen Zheng ${ }^{3, \star}$, Ruozhu Zhao ${ }^{1}$, Zhi-Peng Wang ${ }^{2}$, Wen Shao ${ }^{4}$, Hao-Nan Chang ${ }^{2}$, Jing-Yuan Cheng ${ }^{2}$, Hui Zhao ${ }^{5}$, Lei Liu² \& Hai Qi ${ }^{1}$

Chemokine-guided lymphocyte positioning in tissues is crucial for normal operation of the immune system. Direct, real-time manipulation and measurement of single-cell responses to chemokines is highly desired for investigating the cell biology of lymphocyte migration in vivo. Here we report the development of the first two-photon-activatable chemokine CCL5 through efficient one-pot total chemical synthesis in milligram scale. By spatiotemporally controlled photoactivation, we show at the single-cell level that $T$ cells perceive the directional cue without relying on PI3K activities, which are nonetheless required for persistent migration over an extended period of time. By intravital imaging, we demonstrate artificial T-cell positioning in cutaneous tissues and lymph nodes. This work establishes a general strategy to develop high-quality photo-activatable protein agents through tailor-designed caging of multiple residues and highlights the potential of photo-activatable chemokines for understanding and potential therapeutic manipulation of cell positioning and positioncontrolled cell behaviours in vivo.

\footnotetext{
${ }^{1}$ Tsinghua-Peking Center for Life Sciences, Laboratory of Dynamic Immunobiology, Institute for Immunology, Tsinghua University, Beijing 100084, China. ${ }^{2}$ Tsinghua-Peking Center for Life Sciences, Department of Chemistry, Tsinghua University, Beijing 100084, China. ${ }^{3}$ High Magnetic Field Laboratory, Chinese Academy of Sciences, Hefei 230031, China. ${ }^{4}$ Laboratory for Stem Cells and Epigenetics, School of Medicine, Tsinghua University, Beijing 100084, China. ${ }^{5}$ School of Life Sciences, Tsinghua University, Beijing 100084, China. * These authors contributed equally to this work. Correspondence and requests for materials should be addressed to L.L. (email: Iliu@mail.tsinghua.edu.cn) or H.Q. (email: qihai@mail.tsinghua.edu.cn).
} 
n serving host defense, lymphocytes must patrol potential sites of microbial invasion and rapidly locate locales of unpredictable tissue damage ${ }^{1}$. Chemokines play a key role in these processes, positioning lymphocytes under both quiescent and inflammatory conditions, allowing these migratory cells to interact with distinct microenvironments in spatiotemporally specific manners to develop and execute effector functions ${ }^{2}$. Our understanding of the chemosensing machinery is mainly derived from studies of Dictyostelium and neutrophils, and integrated models of intracellular signalling networks are being assembled to account for chemosensing sensitivity and robustness ${ }^{3}$. However, it is still being debated as to whether signal-centred models fully account for the range of biological behaviours observed with Dictyostelium and neutrophils ${ }^{4,5}$. It is even less clear whether lymphocyte behaviours obey the same paradigm, as different cell types may invoke different mechanisms to sense direction and orchestrate migration. Furthermore, chemokine-mediated recruitment of effector lymphocytes may be exploited to combat infections and tumours. Synthetic chemokines whose activities can be controlled in a spatiotemporally defined manner are a particularly attractive option to direct lymphocyte positioning and recruitment in vivo.

In this study, we have achieved total chemical synthesis and photocaging of human CCL5 (hCCL5) and use this model chemokine to establish a novel approach for studying lymphocyte chemosensing. Our results indicate phosphoinositide-3-kinase (PI3K) is not required for $\mathrm{T}$ cells to sense the directional cue in vitro and provide proof-of-concept demonstration for artificial control of lymphocyte localization in vivo.

\section{Results}

Total synthesis and validation of photoactivatable hCCL5. Mature, bioactive hCCL5 has 68 residues and is chemoattractive towards T cells, basophils and eosinophils through chemokine receptors CCR1, CCR3, CCR4 and CCR5 (ref. 6). To establish a practical procedure for full-length hCCL5 synthesis, we used a one-pot strategy ${ }^{7}$ that is based on 9-fluorenylmethoxycarbonyl (Fmoc) solid-phase peptide synthesis and native chemical ligation using modified thioester equivalents ${ }^{8-10}$. As shown in Fig. 1a, synthetic hCCL5 was made from three consecutive segments (Supplementary Fig. 1), namely $\left[\mathrm{Ser}^{1}-\mathrm{Cys}^{10}\right]-\mathrm{NHNH}_{2}$ (1),

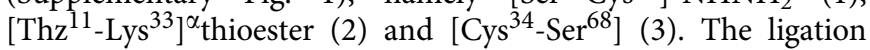
of (2) and (3) in neutral aqueous buffer went to completion in $4 \mathrm{~h}$ to generate $\left[\mathrm{Thz}^{11}-\mathrm{Ser}^{68}\right]$ (4). Addition of $0.25 \mathrm{M}$ methoxyamine $\cdot \mathrm{HCl}$ and $\mathrm{pH}$ adjustment to 4.0 for additional $4 \mathrm{~h}$ resulted in quantitative conversion of (4) to [Cys $\left.{ }^{11}-\mathrm{Ser}^{68}\right]$ (5). After $\mathrm{pH}$ re-adjustment to 6.8 , the same batch was added with a slight excess of $\left[\mathrm{Ser}^{1}-\mathrm{Cys}^{10}\right]^{\alpha}$ thioester, which was prepared by transthioesterification with 4 -mercaptophenyacetic acid of (1). After $2 \mathrm{~h}$, full-length [Ser ${ }^{1}-\mathrm{Ser}^{68}$ ] was generated. The product was purified and folded to afford hCCL5 in an overall isolated yield of $22 \%$ (Supplementary Fig. 2; mass: obsd (mean \pm s.e.m. $=7845.2 \pm 0.7$ ) Da; cald 7844.9 Da, average isotopes). Circular dichroism spectroscopy of the synthetic hCCL5 (Supplementary Fig. 3) was consistent with that of recombinant hCCL5 (ref. 11).

To cage the hCCL5 activity, we chose to modify two serine residues (Ser1 and Ser5) in the amino terminus by 4,5-dimethoxy-2-nitrobenzyl (DMNB) chemistry. Previous mutagenesis studies indicated that these residues are important for receptor binding and activation ${ }^{12}$. Two singly caged analogues hCCL5 $\left(\mathrm{S}_{1} \mathrm{DMNB}\right.$ ) (mass: obsd (mean \pm s.e.m. $=8040.4 \pm 0.8$ ) $\mathrm{Da}$; cald $8039.9 \mathrm{Da}$, average isotopes) and hCCL5( $\left.{ }_{5} \mathrm{DMNB}\right)$ (mass: obsd (mean \pm s.e.m. $=8040.3 \pm 0.3$ ) Da; cald $8039.9 \mathrm{Da}$, average isotopes), and a doubly caged analogue hCCL5
$\left(\mathrm{S}_{1,5} \mathrm{DMNB}\right)$ (mass: obsd (mean \pm s.e.m. $\left.=8235.1 \pm 0.5\right) \mathrm{Da}$; cald 8235.0 Da, average isotopes) were successfully obtained using the same synthetic route as described above (Fig. 1a and Supplementary Fig. 2). Circular dichroism spectra of all the caged proteins showed a negative minimum at $203 \mathrm{~nm}$, revealing that attachment of photosensitive DMNB groups to the $\mathrm{N}$-terminal serine(s) did not appreciably change the overall hCCL5 structure (Supplementary Fig. 3). We then tested bioactivity of these synthetic hCCL5 proteins in a transwell migration assay using murine $\mathrm{CD}^{+}{ }^{+} \mathrm{T}$ lymphocytes, which migrated towards hCCL5 in a CCR5-dependent manner (Supplementary Fig. 4). Whereas our synthetic native hCCL5 (Fig. 1b) exhibits activities indistinguishable from that of recombinant hCCL5 (not shown), chemotactic activities of the two singly caged forms were reduced but not abrogated (Fig. 1b). On the other hand, the doubly caged hCCL5 protein exhibited no measurable chemotactic activity. On exposure to the ultraviolet light $\left(365 \mathrm{~nm}, 18 \mathrm{~mW} \mathrm{~cm}^{-2}\right)$ for $5 \mathrm{~min}$, the photolytic release of DMNB was complete (Supplementary Fig. 5a) and full chemotactic activity was restored for all the three caged analogues (Fig. 1b). Therefore, DMNB caging of both Ser 1 and Ser 5 generates fully caged and rapidly photoactivatable hCCL5. It is noteworthy that backbone caging was previously attempted to create a photoactivatable chemokine, but its uncaging required ultraviolet illumination for at least $60 \mathrm{~min}^{13}$, a process much slower than chemokine-regulated cell behaviours by orders of magnitude. By caging the chemokine-receptor interaction interface, our study establishes a general strategy to control chemokine activities with a very high temporal resolution.

With a practical uncaging method in hand, we recognized that illumination by the ultraviolet light is cytotoxic and unable to penetrate deep biological tissues. Intravital two-photon microscopy, owing to reduced photo-toxicities and tissue scattering of its long-wavelength excitation light, is particularly suited for studying immune cell migration and positioning dynamics in vivo ${ }^{94}$. Therefore, we tried caging of hCCL5 with a twophoton-sensitive protective group, nitrodibenzofuran (NDBF). NDBF exhibits excellent two-photon photochemical properties (two-photon cross-section $=0.6 \mathrm{GM}$ at $720 \mathrm{~nm}$, half-time for diffraction-limited two-photon photolysis $=0.3 \mathrm{~ms}$ ) and has been successfully used to control $\mathrm{Ca}^{2}+$ release from the sarcoplasmic recticulum of intact cardiac myocytes ${ }^{15}$. We prepared FmocSer(NDBF)-OH (Supplementary Fig. 6 and Supplementary Methods) and, by Fmoc solid-phase peptide synthesis, obtained $\left[\operatorname{Ser}^{1}-\mathrm{Cys}^{10}\left(\operatorname{Ser}_{1,5} \mathrm{NDBF}\right)\right]-\mathrm{NHNH}_{2}$. By the same synthetic strategy established in Fig. 1a, doubly NDBF-caged hCCL5 (denoted as hCCL5 $5^{\star *}$ ) was successfully synthesized in multimilligram scale (13\% isolated yield, Fig. 1c,d and Supplementary Fig. 7) (mass: obsd (mean \pm s.e.m. $=8322.6 \pm 0.3$ ) Da; cald 8323.0 Da, average isotopes). Analysis of photolytic kinetics by the $365-\mathrm{nm}$ ultraviolet light revealed that hCCL5 $5^{\star *}$ uncaging was completed within $30 \mathrm{~s}$, which was almost ten times faster than that with DMNB (Supplementary Fig. 5b). Transwell assay confirmed that hCCL $5^{\star \star}$ had essentially no chemotactic activity towards $\mathrm{CD}^{+}{ }^{+} \mathrm{T}$ cells before ultraviolet illumination, but fully recovered the activity on irradiation $\left(365 \mathrm{~nm}, 18 \mathrm{~mW} \mathrm{~cm}^{-2}\right)$ for $30 \mathrm{~s}$ (Fig. 1e).

Next, we tested two-photon uncaging of hCCL $5^{\star *}$ using video microscopy. As shown in Fig. 2a and Supplementary Movie 1, a $\mathrm{CD}^{+}{ }^{+} \mathrm{T}$ cell placed in media containing hCCL5 ${ }^{\star *}$ on the ICAM-2-coated plate was guided by the focus of a 720 -nm laser as it was manually moved across the field. Complex patterns of T-cell localization can be achieved with freely definable uncaging light paths, as exemplified by the heart-shaped configuration in Fig. $2 \mathrm{~b}$ and Supplementary Movie 2. In addition, to test the 
a

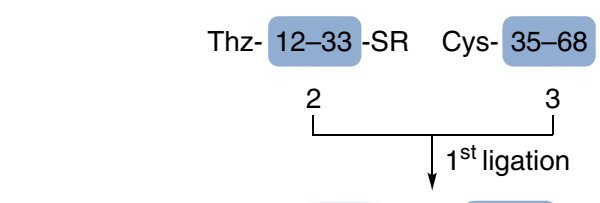

Thz- 12-33 -Cys - 35-68

4

?

1-10 $-\mathrm{NHNH}_{2}$ Cys- 12-33 -Cys- 35-68

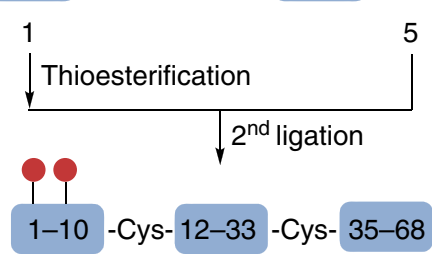

Full-length hCCL5

Refolding

Thz-to-Cys

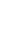 \\ (}



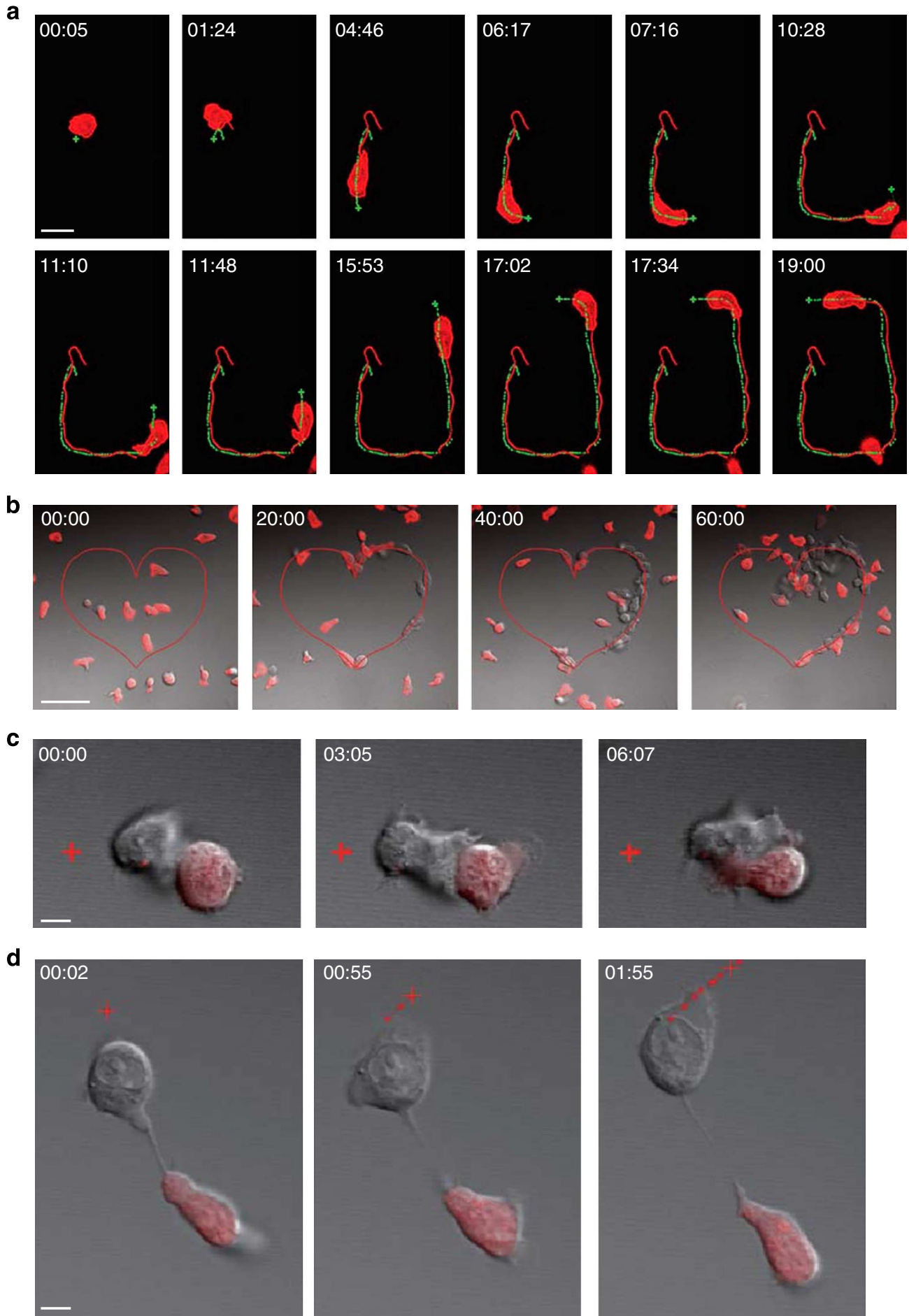

Figure 2 | Two-photon guided T-cell migration in vitro. (a) Time-lapse images of a dsRed-expressing CD8 ${ }^{+}$T cell chasing the focus of a 720 -nm twophoton excitation laser in media containing $1 \mu \mathrm{g} \mathrm{ml} \mathrm{m}^{-1} \mathrm{hCCL} 5^{\star \star}$. The uncaging laser focus was manually placed in front of the leading edge. Green dots: previous positions of the laser focus; green cross: current focus at the indicated time point; red dots: centroid positions of the cell. Scale bar, $20 \mu \mathrm{m}$.

(b) Time-lapse images showing T-cell accumulation over time towards the uncaging light path patterned in a heart shape (red line) in hCCL5 ${ }^{\star \star}$-containing media. Scale bar, $50 \mu \mathrm{m}$. Data representative of more than five independent experiments. Time-lapse images showing a stable (c) or unstable (d) contact engaged CD8 + T-cell response to hCCL5 attraction delivered by a focus of 720-nm two-photon excitation continuously. Red dots: previous positions of the laser focus; red cross: current focus at the indicated time point. Scale bar, $5 \mu \mathrm{m}$. Data are representative of more than five independent experiments.

cytoskeleton rearrangement organized by T-cell receptor signalling and recruitment of chemokine receptors, probably CCR5, to the immunological synapse (Supplementary Fig. 4 and ref. 3). Therefore, by taking advantage of the rapid two-photon uncaging kinetics and developing the first activity-caged, two-photon- activatable chemokine, we have created a new way to deliver sitespecific chemokine stimulation and manipulate directional T-cell migration in real time. A key aspect of this technology is the high efficiency and flexibility of the chemical synthesis scheme, which provides convenient access to tailor-designed caged proteins 
bearing modifications at multiple sites. More importantly, the synthetic method described here can be used to develop other activity-caged chemokines and other small- to medium-sized proteins for mechanistic and therapeutic studies.

PI3K is required for migration but not directional sensing. A photo-activatable chemokine can be an excellent tool to study spatiotemporally controlled cell behaviours at the cellular level as well as signalling transduction at high spatiotemporal resolution at the molecular level. As a demonstration, we examined the role of PI3K in T-cell chemotaxis in vitro. Earlier studies of Dictyostelium and neutrophil migration indicate that PI3K is essential for sensing the chemokine gradient and driving directional migra$\operatorname{tion}^{16-19}$. More recent work, however, suggests that Dictyostelium cells or neutrophils deficient in PI3K activities are still capable of directional migration to certain extent, even though they exhibit reduced polarization, speed and fraction of responding cells ${ }^{20,21}$. Precisely, for what purpose PI3K is required during the process of eukaryotic chemotaxis is not fully understood ${ }^{22}$. Lymphocytes have rarely been studied in this regard, in part because of their small sizes and associated difficulties to manipulate, despite the fundamental importance of chemotaxis for their normal functions. A recent in-vivo study demonstrates that during the induction of a germinal centre response, migration of $\mathrm{T}$ lymphocytes from the T-cell zone into the B-cell follicle requires not only the chemokine receptor CXCR5 but also the inducible co-stimulator receptor that independently activates PI $3 \mathrm{~K}^{23}$, further raising the question as to how PI3K activities not associated with the guidance receptor per se contribute to the directional migration of $\mathrm{T}$ lymphocytes. We thus further explored the role of PI3K in T-cell chemotaxis in vitro using the newly established system. As shown in Fig. 3a (time 0), when T cells were briefly treated with $100 \mathrm{nM}$ wortmannin, a majority of them became depolarized (the ratio between long and short axes of the cell, or shape index, $<1.5$ ), as can be expected ${ }^{24}$. However, these cells were still able to migrate, albeit with much reduced speeds, up to the CCL5 gradient and eventually accumulated at the source of active CCL5 to a significant degree (Fig. 3a and Supplementary Movie 5 and 6). These data suggest that similar to Dictyostelium cells or neutrophils ${ }^{20,21}$, $\mathrm{T}$ lymphocytes do not absolutely need PI3K activities for directional migration. However, at the population level it was difficult to determine whether this is because PI3K-inhibited T cells could not perceive the gradient as effectively or migrate as efficiently, or both. Therefore, to probe whether, beyond cellular polarization and maintaining migration speed, PI3K is required for additional processes involved in chemotaxis, we took advantage of the unique ability of our system to create a point source $\left(<0.1 \mu \mathrm{m}^{2}\right)$ of bioactive chemokine, owing to the small volume of effective two-photon excitation and examined one-by-one behaviours of individual $\mathrm{T}$ cells that are polarized (shape index $>1.5$ ) despite PI3K inhibition. As shown in the diagram of Fig. 3b, we placed the point source by the side of the $\mathrm{T}$ cell roughly at the middle point between the front and the back and video-recorded cell migration to determine how fast a $\mathrm{T}$ cell could turn $90^{\circ}$ and points its front towards the CCL5 point source. Three types of pseudopod behaviours were observed: some cells immediately generated a new front facing the point source $(\mathrm{N}$-turn behaviour; Fig. 3c and Supplementary Movie 7) or extended its existing pseudopod front towards the point source and make a sharp turn (U-turn behaviour; Fig. 3d and Supplementary Movie 8), whereas additional cells exhibited significant 'hesitation', alternatively extending pseudopods towards and away from the source for a significant period of time (C-turn behaviour; Fig. $3 \mathrm{e}$ and Supplementary Movie 9). Some $\mathrm{T}$ cells exhibited a hybrid response of mixed $\mathrm{N}$ - and C-turn (mix-turn behaviour; Supplementary Fig. 8). We calculated $\cos (\theta)$ every $10 \mathrm{~s}$ for each cell and plotted it against time. The amount of time it takes for $\cos (\theta)$ to decay from 1 to 0 is a universal measurement for how effectively the cell senses and executes the change of direction (Fig. 3b). As shown in Fig. 3f and Supplementary Fig. 8, wortmannin-treated cells changed directions as efficiently as the untreated cells in the first $30 \mathrm{~s}$ after chemokine exposure, but beyond this short period, even when the cell shape polarization was apparently normal, PI3K-inhibited cells were less efficient in following the point source of CCL5. These data suggest that in T lymphocytes, PI3K activities are not responsible for sensing the direction per se but for coordination of persistent movement to execute the migration towards the sensed direction. To test whether an increase in the strength of the CCL5 gradient could ameliorate the defect, we increased the power of the uncaging laser by eightfold. As shown in Fig. 3g, the decay of $\cos (\theta)$ was indeed accelerated in the stronger field for both control and wortmannin-treated cells; however, the defect beyond $30 \mathrm{~s}$ remained unchanged. Therefore, beyond promoting cell polarization, PI3K is required for persistent migration but not necessarily for direction sensing per se.

Light-guided migration and relocation of $\mathrm{T}$ cells in vivo. When combined with intravital microscopy, a two-photon caged chemokine system could offer the ability to deliberately localize migratory immune cells to desired location in vivo. To test this, we first conducted ear skin imaging following intradermal injection of hCCL5** and green fluorescent protein (GFP)expressing $\mathrm{CD} 8{ }^{+} \mathrm{T}$ cells activated in vitro. Similar cell transfer protocols have been used to probe behaviours of neutrophils in inflamed tissues ${ }^{25}$. Typically within $1 \mathrm{~h}$ after transfer, $\mathrm{CD}^{+}$ $\mathrm{T}$ cells would settle in and migrate in an apparently random manner in the dermal tissue. We used a dual-laser system in which a pulsed $920-\mathrm{nm}$ laser was used to image the $\mathrm{GFP}^{+} \mathrm{T}$ cells, while a second pulsed 720-nm laser was used to photo-stimulate a pre-defined cylindrical volume of interest (VOI). As shown in Fig. $4 \mathrm{a}$ and Supplementary Movie 10 , the $\mathrm{CD} 8{ }^{+} \mathrm{T}$ cells were seen to stream into the VOI soon after the onset of uncaging, while these cells remained randomly migrating when PBS instead of hCCL5 ${ }^{* *}$ was injected. To quantitatively characterize the directionality of $\mathrm{CD}^{+} \mathrm{T}$ cells migrating towards the VOI source of active hCCL5, we defined the chemotactic index (CI) as the cosine of the angle between the vector connecting the cell to the VOI centre and the vector of the cell instantaneous velocity (Fig. 4b). Before uncaging, the transferred T cells migrated with an average $\mathrm{CI}$ of 0.03 . After uncaging, the average $\mathrm{CI}$ rose to 0.19 during the first $10 \mathrm{~min}$, with a significant number of cells exhibiting individual CIs close to 1 . In contrast, the control group exhibited an average of 0.01 (Fig. 4c). We also observed a relationship between the strength of chemotactic behaviours and the distance from the chemokine source. As shown in Fig. 4d, within the VOI itself $(0-20 \mu \mathrm{m}$ to the centre), cells exhibited an average $\mathrm{CI}$ of 0 , consistent with the fact that cells do not show directionality in a uniform field of any chemokine. The CI increased to $\sim 0.4$ within the belt $40-60 \mu \mathrm{m}$ away from the centre and rapidly decreased farther away. At the end of the 50-min imaging and photo-stimulation, the average cell number within the VOI rose from 3 to 8 . These data demonstrate a chemotactic gradient can be artificially established in vivo through two-photon uncaging of a caged chemokine to acutely relocalize lymphocytes. Finally, we further attempted to manipulate lymphocyte positioning in the lymph node, a critical organ in which many of the fate decisions during lymphocyte effector development are made in a spatiotemporally 
a
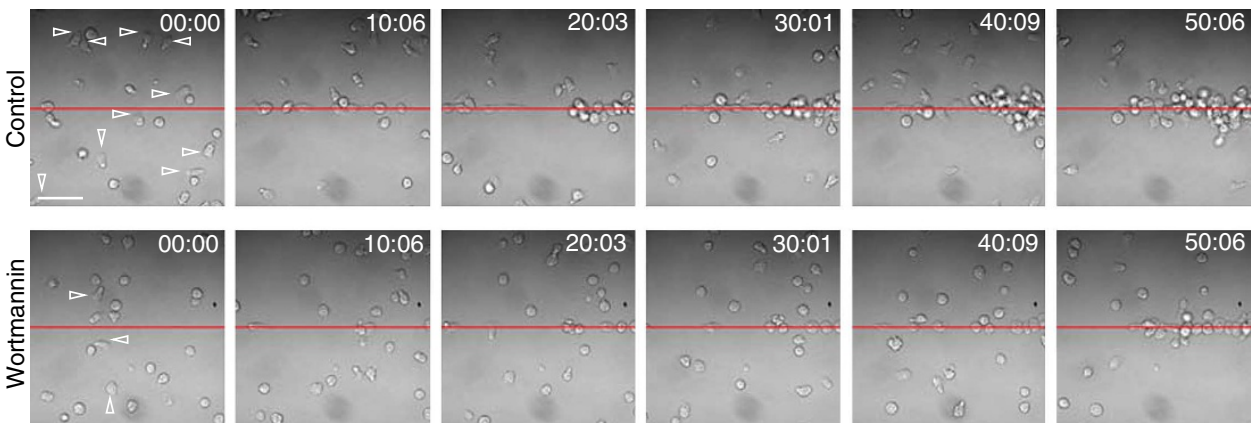

b

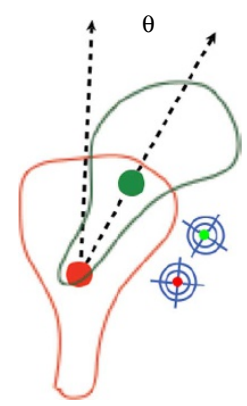

e
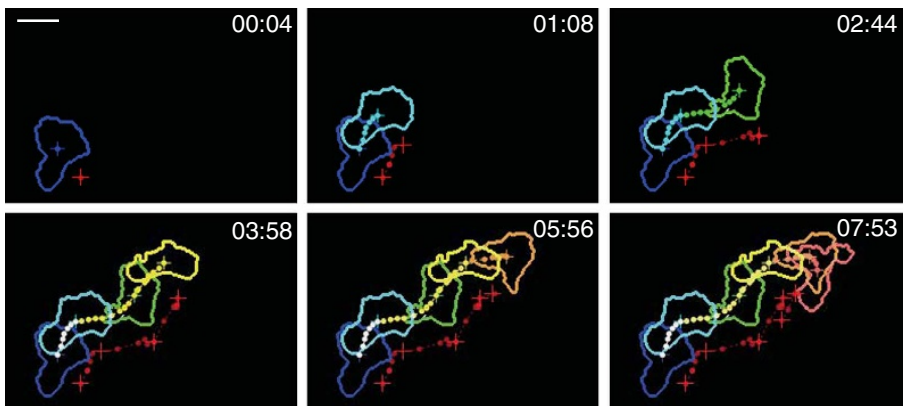

C
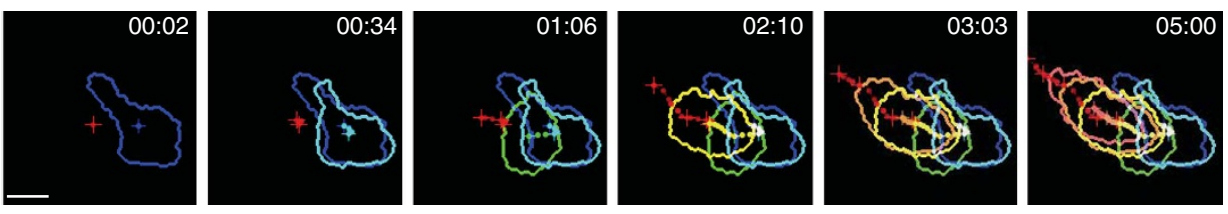

d
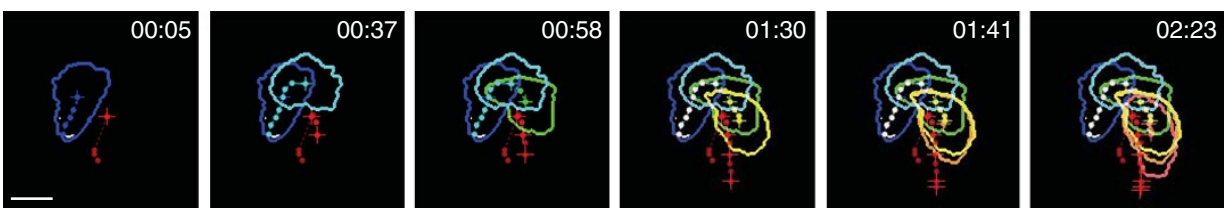

f
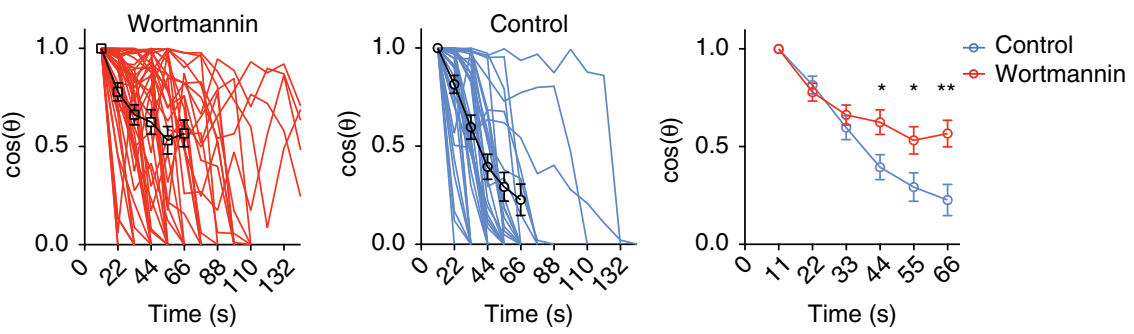

g
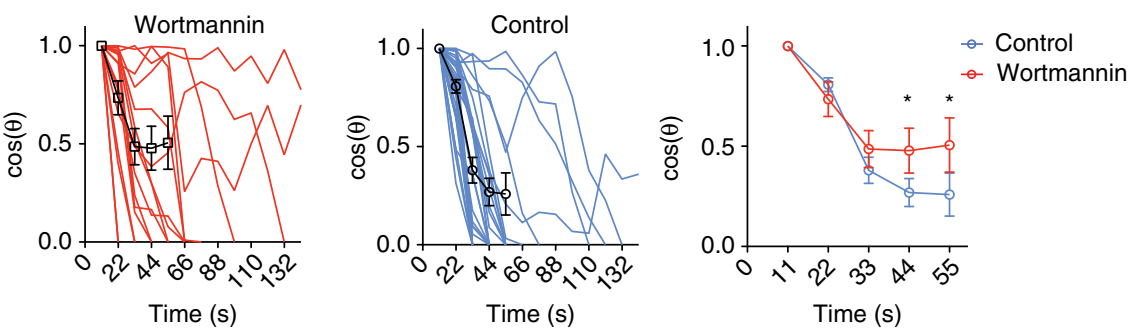

Figure 3 | PI3K activities required for persistent migration but not directional sensing. (a) Time-lapse images showing directed migration and accumulation of T cells, untreated or treated with $100 \mathrm{nM}$ wortmannin, in $\mathrm{hCCL} 5^{\star \star}$-containing media towards the uncaging light path patterned as a line. Open arrowheads: polarized cells with a shape index $>1.5$. Scale bar, $50 \mu \mathrm{m}$. (b) Schematic diagram of single T-cell chemotaxis assay by two-photon uncaging of hCCL5**. The focal point $\left(\sim 0.078 \mu \mathrm{m}^{2}\right.$, crossed circles) always placed on one side of the cell. Angle $\theta$ is between the current instantaneous velocity vector and the velocity vector at the first time point after uncaging. The centroid positions of the cell are denoted with the red and green dots. Time-lapse images of T cells that, in response to active CCL5, generate new pseudopods (c, ' $\mathrm{N}$-turn') or extend existing pseudopods towards the uncaging light focus ( $\mathbf{d}$, 'U-turn') or remain uncommitted for a prolonged period (e, 'C-turn'); cell shapes outlined in colours of different warmth to indicate time and pseudopod dynamics. See corresponding Supplementary Movie 7-9. Scale bar, $10 \mu \mathrm{m}$. (f,g) Kinetic decay curves of cosine $(\theta)$. For each cell, values of $\operatorname{cosine}(\theta)$ before it reaches zero were used in calculation. Cells whose cosine $(\theta)$ remained positive throughout the intial $200 \mathrm{~s}$ on uncaging were excluded. T cells untreated or treated with wortmannin $(100 \mathrm{nM})$ at $10 \%(\mathbf{f})$ or $80 \%$ laser power output for uncaging ( $\mathbf{g}$ ); left and middle: curves for individual cells (coloured) and mean \pm s.e.m. (black); right: comparison of untreated and treated; ${ }^{\star} P<0.05,{ }^{\star \star} P<0.01$, by two-tailed $t$-tests. 
a

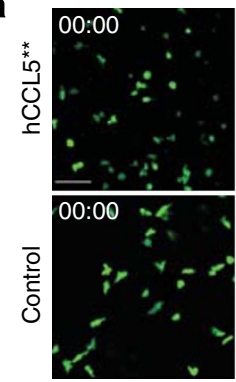

b

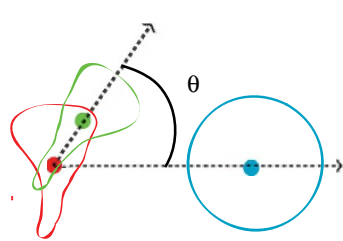

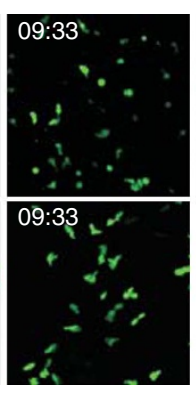
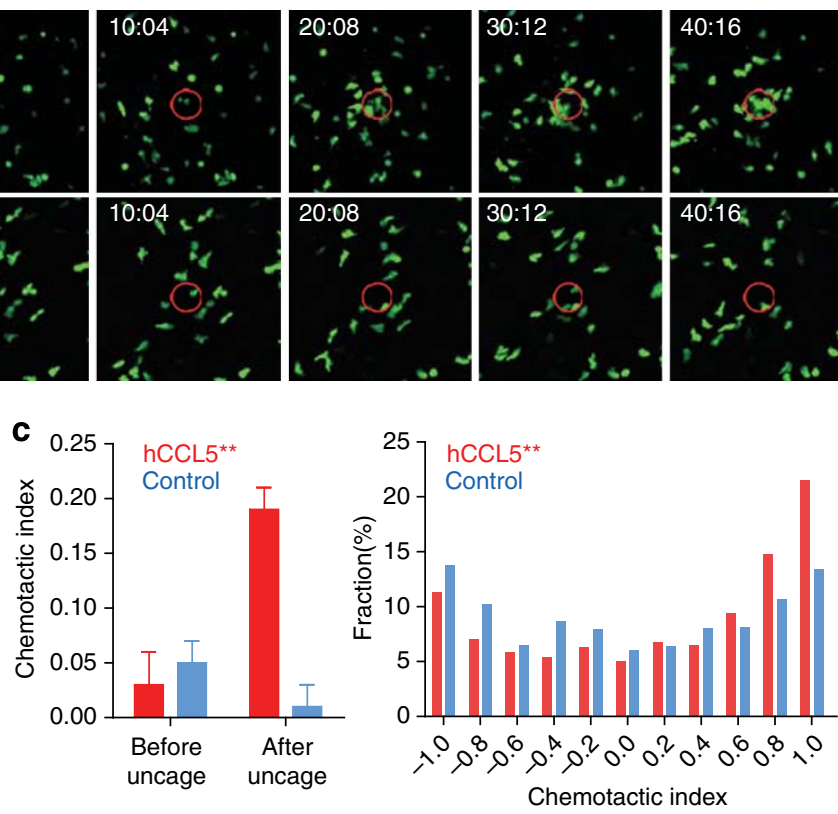

Chemotactic index
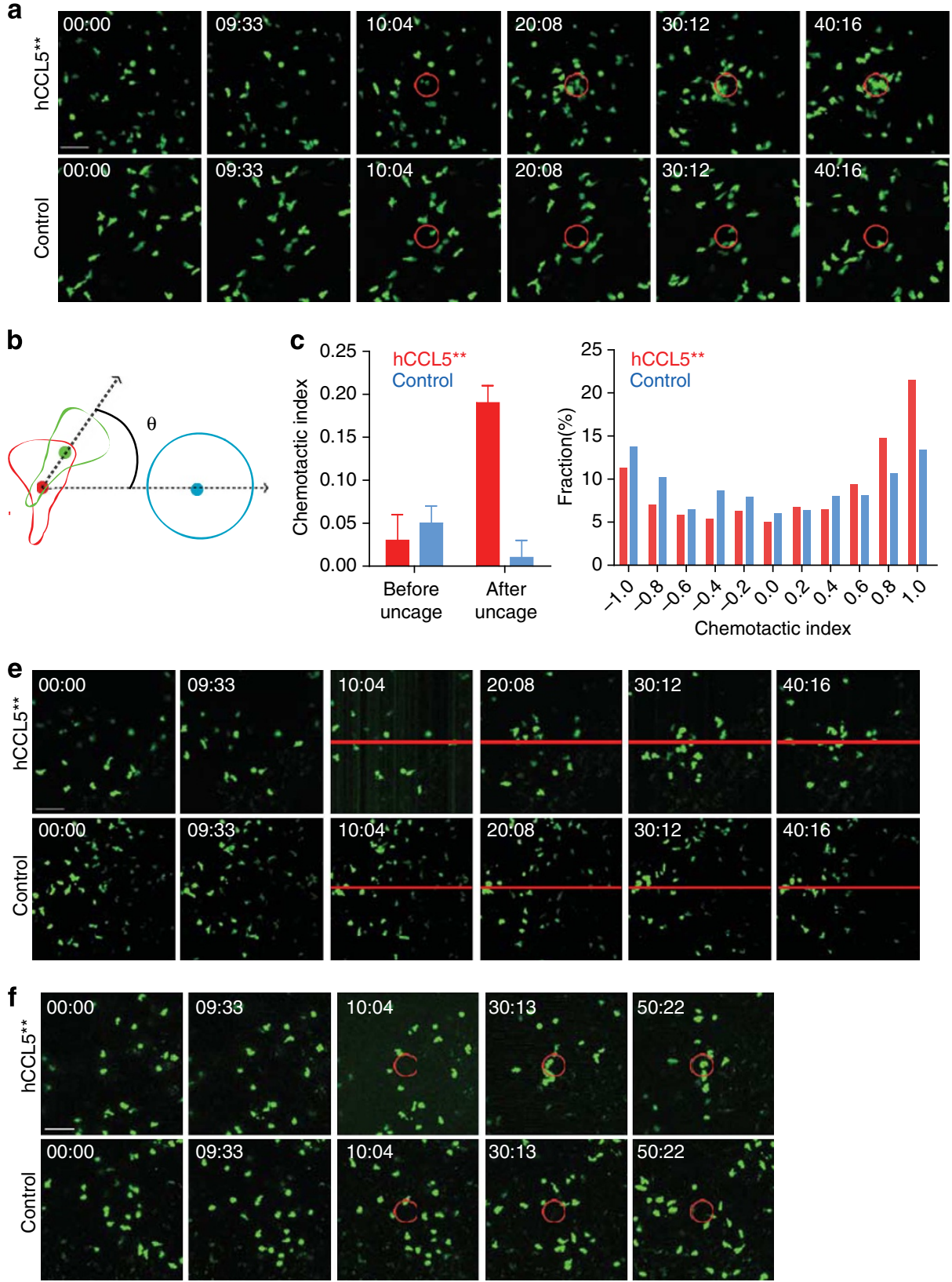
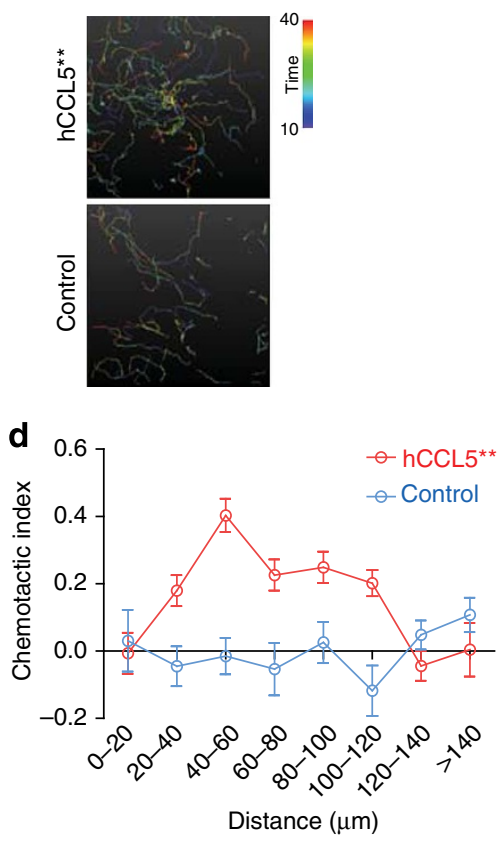

Figure 4 | Light-guided migration and relocation of T cells in vivo. (a-d) Uncaging of hCCL5*^ and directional T-cell migration in dermal tissues. (a) Timelapse images (left) and time-coded tracks (right) of T cells migrating in dermal tissues of the ear injected with hCCL5 ${ }^{\star \star}$ or PBS (control) before and after uncaging by raster-scanning with 720-nm light in the marked area (red circle). Cell tracks during the first 20 min immediately after the onset of uncaging. Scale bar, $50 \mu \mathrm{m}$. (b) Definition of the chemotactic index $(\mathrm{Cl})$ as the cosine of the angle $\theta$ between the velocity vector and the vector connecting the cell to the VOI centre. (c) Left, average $\mathrm{Cl}$ of control and hCCL5 ${ }^{\star \star}$ group 10 min before and after uncaging; right, $\mathrm{Cl}$ distribution of cells starting from outside of the VOI during the first $10 \mathrm{~min}$ immediately after the onset of uncaging. (d) Cls plotted against distances away from the centre of the VOI as in $\mathbf{a}$. (e, $\mathbf{f}$ )

Directional T-cell migration in the lymph node after uncaging hCCL5 $5^{\star \star}$ either by a light path patterned as a line (e) or as a circle (f) on all optical sections (3- $\mu \mathrm{m}$ step). Scale bar, $50 \mu \mathrm{m}$. Error bars indicate s.e.m.

defined manner. To this end, we adoptively transferred GFPexpressing $\mathrm{CD}^{+} \mathrm{T}$ cells intravenously and injected hCCL5 ${ }^{* *}$ intravenously during the imaging session. As shown in Fig. 4e and Supplementary Movie 11, when uncaging was conducted as a 'line', essentially a sheet in the $z$ direction of the three-dimensional space, directed migration and accumulation of T cells towards the 'line' were observed. When uncaging was done within a cylinder VOI that was as deep as $100-150 \mu \mathrm{m}$ below the capsule, $\mathrm{T}$ cells were also seen to collectively migrate towards the VOI, with some cells migrating from tissue regions above the upper bound of or below the lower bound of the imaging stack (Fig. 4f and Supplementary Movie 12). Together, these data demonstrate creation of artificial chemotactic fields and on-demand repositioning of lymphocytes in vivo.

\section{Discussion}

In this study, we have created a photo-caged chemokine that allows highly efficient, real-time two-photon uncaging in vitro and in vivo. Chemokines activatable by two-photon illumination represents a novel strategy to study biochemistry and cell biology of cell migration. A distinct advantage is the high spatiotemporal 
precision it offers. As demonstrated in our study, on very short $(<5 \mathrm{~s})$ and focused $\left(<0.1 \mu \mathrm{m}^{2}\right) 720-\mathrm{nm}$ two-photon irradiation, uncaged hCCL $5^{* *}$ induces pseudopod dynamics in $\mathrm{CD} 8^{+} \mathrm{T}$ lymphocytes, in principle enabling studies of chemokinetriggered signalling events inside single pseudopod with a temporal resolution of seconds. Another important advantage of the new system is that it can be used in vivo when combined with intravital two-photon microscopy. Indeed, using the twophoton-activatable hCCL5 $5^{* *}$, we have demonstrated its application in artificial lymphocyte repositioning in vivo, both in dermal tissues and deep inside the lymph node. The in-vitro findings that $\mathrm{PI} 3 \mathrm{~K}$ is not required for direction sensing per se but for persistent migration of lymphocytes, as revealed here at the single-cell level by the new experimental system, are in good agreement with observations in vivo that PI $3 \mathrm{~K}$ activities continuously triggered by non-G-protein-coupled-receptor receptors facilitate chemokineguided directional migration by promoting persistent motility ${ }^{23}$, and support a more pseudopod-centred view than a strict compass-based model of chemotaxis ${ }^{3,4,22}$. On the other hand, much faster imaging instrument has to be developed to take full advantage of the spatiotemporal controllability offered by the caged agent to study such single-cell kinetic responses to chemokines in the true three-dimentional tissue space in vivo. Our work also suggests potential application of two-photonactivatable chemokines in studies of chemokine signalling during synapse/kinapse formation. Therefore, our caged chemokine system, by allowing artificial cell repositioning, opens new avenues for studying immune cell dynamics and spatiotemporal regulation of cell fate determination in vivo.

Our results indicate that caging of more than one amino acid residues is needed to develop a tightly controlled photo-activatable protein agent. Whereas incorporation of multiple tailor-designed amino acids is difficult to accomplish with recombinant engineering technology, direct chemical synthesis ${ }^{26}$ is particularly suited for such tasks. The activity-caging strategy presented in this work is to modify key residues of the CCL5 chemokine ligand-receptor interaction interface. To generalize the strategy to any chemokines or other types of protein ligands, the only information needed is molecular details of the protein-protein interaction interface obtainable by either alanine scan mutagenesis or crystal structure analysis. For small- to medium-sized proteins or protein domains (for example, <200 amino acid), technology demonstrated here should enable synthesis in the multi-milligram scale, sufficient for both in-vitro and in-vivo studies.

Intercellular communication among different cell types through secreted factors such as chemokines, cytokines and hormones represents one of the most sophisticated and complex aspects of tissue physiology. The strategy of synthetic chemistry combined with a live-imaging approach, as developed and adopted in the present study, demonstrates a novel platform to interrogate responses of single cells to any protein factors in physiological tissue environment in a spatiotemporally defined manner.

\footnotetext{
Methods

Peptide synthesis. All peptides were manually synthesized using standard Fmoc protocols. The 2-Cl-(Trt)- $\mathrm{NHNH}_{2}$ resin was prepared directly from $2-\mathrm{Cl}$-(Trt)-Cl resin by treatment with $5 \% \mathrm{NH}_{2} \mathrm{NH}_{2}$ in $\mathrm{N}, \mathrm{N}$-dimethylformamide (DMF) two times, $30 \mathrm{~min}$ for each time to prepare the corresponding peptide hydrazides. An enamide motif ${ }^{27}$ attached to Rink Amide resin was used for peptide thioester synthesis. For each coupling reaction, the resin was coupled with amino acid (4 eq.), 2-(6-chloro-1H-benzotriazole-1-yl)-1,1,3,3-tetramethylaminiumhexafluorophosphate (HCTU)/1-[bis(dimethylamino)methylene]-1H-1,2,3-triazolo[4,5-b]pyridinium hexafluorophosphate 3-oxide (HATU) (3.6 eq.) in $0.25 \mathrm{M}$ $\mathrm{N}, \mathrm{N}$-diisopropylethylamine (DIEA) solution. Proper coupling conditions were chosen for each amino acid, including the number of coupling reactions (for example, single or double coupling) and the type of coupling reagent (HCTU/ HATU). A single-coupling reaction using HCTU for $60 \mathrm{~min}$ was enough for most of the amino acids. For sterically hindered amino acids (such as Fmoc-Asn(Trt)-
}

$\mathrm{OH}$, Fmoc-Ile-OH, Fmoc-Gln(Trt)-OH, Fmoc-Arg(Pbf)-OH, Fmoc-Val-OH and Fmoc-Thr(tBu)-OH) and the amino acid right after Pro, a double-coupling strategy was recommended. For Fmoc-Ser(DMNB)-OH and Fmoc-Ser(NDBF)-OH, the photo-sensitive amino acid (1.5 eq.), HATU (1.5 eq.) and HOAt (1.5 eq.) in $0.5 \mathrm{M}$ DIEA/DMF solution was used for coupling. Twenty per cent piperidine in DMF (v/ v) was used to remove the Fmoc protecting group. The overall deprotection of sidechain protecting groups and final cleavage from the resin were achieved by trifluoroacetic acid cocktails ( $88 \%$ trifluoroacetic acid, $5 \%$ phenol, $2 \%$ triisopropylsilane, $5 \% \mathrm{H}_{2} \mathrm{O}$ ) for about $2 \mathrm{~h}$ at room temperature (r.t.). The combined solutions were concentrated by blowing with $\mathrm{N}_{2}$ and then treated with cold dry ether. The crude peptides obtained after centrifugation were dissolved in $\mathrm{CH}_{3} \mathrm{CN} /$ $\mathrm{H}_{2} \mathrm{O}$ for further analysis and purification. Peptides were confirmed by matrix assisted laser desorption/ionization-time-of-flight-mass spectrometry (MS) or electrospray ionization-MS.

A one-pot synthesis of hCCL5 and its caged analogues.. (A) Native chemical ligation of $\left[\mathrm{Thz}^{11}-\mathrm{Lys}^{33}\right]^{\alpha}$ thioester and $\left[\mathrm{Cys}^{34}-\mathrm{Ser}{ }^{68}\right]$ and Thz-to-Cys conversion: $\left[\mathrm{Thz}^{11} \text {-Lys }^{33}\right]^{\alpha}$ thioester (1.0 eq.) and $\left[\mathrm{Cys}^{34}{ }^{-S^{2} \mathrm{~S}^{68}}\right.$ ] (1.0 eq.) were dissolved in ligation buffer $\left(6 \mathrm{M} \mathrm{Gn} \cdot \mathrm{HCl}, 200 \mathrm{mM} \mathrm{Na} 2 \mathrm{HPO}_{4}, 100 \mathrm{mM}\right.$ 4-mercaptophenylacetic acid (MPAA), $20 \mathrm{mM}$ tris(2-carboxyethyl)phosphine hydrochloride (TCEP), $\mathrm{pH}$ 6.8), to reach a final concentration of about $4 \mathrm{mM}$. The resulting solution was stirred at r.t. for $4 \mathrm{~h}$. Solid $\mathrm{MeONH}_{2} \cdot \mathrm{HCl}$ (with the final concentration of $0.25 \mathrm{M}$ ) was added and the $\mathrm{pH}$ was adjusted using $6 \mathrm{M} \mathrm{HCl}$ to about 4.0. The reaction was continued for another $4 \mathrm{~h}$ to convert Thz-peptide to Cys-peptide.

(B) Oxidation and thioesterification of $\left[\mathrm{Ser}^{1}-\mathrm{Cys}^{10}\right]-\mathrm{NHNH}_{2}$ and its caged forms: Each peptide hydrazide (1.2 eq.) was dissolved at a concentration of $4 \mathrm{mM}$ in $0.2 \mathrm{M}$ phosphate solution containing $6 \mathrm{M} \mathrm{Gn} \cdot \mathrm{HCl}, \mathrm{pH} 3.0$. The solution was placed in a $-15^{\circ} \mathrm{C}$ ice-salt bath and gently agitated by magnetic stirring. Ten eq. $\mathrm{NaNO}_{2}(0.5 \mathrm{M})$ to the peptide was added to oxidize the peptide hydrazide to azide within $20 \mathrm{~min}$. Subsequently, $200 \mathrm{mM}$ MPAA (dissolved in $0.2 \mathrm{M}$ phosphate solution containing $6 \mathrm{M} \mathrm{Gn} \cdot \mathrm{HCl}, \mathrm{pH}$ 6.0) with equal volume was pipetted to the reaction to eliminate excess $\mathrm{NaNO}_{2}$ and convert the peptide azide to the corresponding peptide thioester. The reaction was then removed from the ice-salt bath and kept at r.t. for $10 \mathrm{~min}$. It is noteworthy that on the condition of [Ser ${ }^{1-}$ $\left.\operatorname{Cys}^{10}\left(\operatorname{Ser}_{1,5} \mathrm{DMNB}\right)\right]-$ MPAA and $\left[\operatorname{Ser}^{1}{ }_{-} \mathrm{Cys}^{10}\left(\operatorname{Ser}_{1,5} \mathrm{NDBF}\right)\right]-\mathrm{MPAA}, 20 \%(\mathrm{v} / \mathrm{v})$, 2,2,2-trifluoroethanol (TFE) was added to solve the solubility problem. The thioester of Segment $1 \mathrm{can}$ also be isolated and lyophilized for the second ligation.

(C) Native chemical ligation of $\left[\mathrm{Ser}^{1}-\mathrm{Cys}^{10}\right]-\mathrm{MPAA}$ (or its caged forms) and $\left[\mathrm{Cys}^{11}{ }^{1}-\mathrm{Ser}^{68}\right.$ ]: Solution containing [Ser ${ }^{1}-\mathrm{Cys}^{10}$ ]-MPAA (or its caged forms) was entirely added to the reaction mixture containing $\left[\mathrm{Cys}^{11}-\mathrm{Ser}^{68}\right]$ and the $\mathrm{pH}$ was adjusted to 6.8. This mixture was continued to react for another $2 \mathrm{~h}$, to generate to full-length hCCL5 (or its caged forms). The whole process was monitored by liquid chromatography-MS. The final product was purified by semi-preparative HPLC and lyophilized.

Refolding of hCCL5 and its caged analogues. Purified hCCL5 and its caged analogues were dissolved in an aqueous buffer of $2 \mathrm{M} \mathrm{Gn} \cdot \mathrm{HCl}$ and $100 \mathrm{mM}$ Tris containing $8 \mathrm{mM}$ cysteine and $1 \mathrm{mM}$ cystine, $\mathrm{pH}$ 8.0. The solutions were gently stirred overnight at r.t., purified by semi-preparative HPLC and confirmed by electrospray ionization-MS.

Ultraviolet photolysis of caged peptides. Photolysis by ultraviolet irradiation was performed on Omnicure S1500 (EXFO Photonic Solutions Inc., Canada) with a $365-\mathrm{nm}$ filter, at a light intensity of $18 \mathrm{~mW} \mathrm{~cm}^{-2}$. Purified peptides with photosensitive groups were dissolved in phosphate buffer containing $6.0 \mathrm{M} \mathrm{Gn} \cdot \mathrm{HCl}, \mathrm{pH}$ 7.4. Analytical HPLC was used to analyse the results of irradiation with varying lengths of time: $0,5,10,30,60,120,180,240$ and $300 \mathrm{~s}$. Benzyl amide as the internal reference standard was used for quantification of reactant and product.

Mice. B6 (Jax 664), albino B6 (Jax 058), GFP-expressing B6 (Jax 4353), dsRedexpressing B6 (Jax 6051) and CCR5 ${ }^{-1-}$ (Jax 5427) mice were from the Jackson Laboratory. OVA $257-264$-specific T-cell receptor transgenic OT-I (Jax003831) was a gift from Dr Yan Shi in Tsinghua University. All mice were maintained under specific pathogen-free conditions and all animal protocols were approved by the Tsinghua Animal Care and Usage Committee in accordance of governmental and institutional guidelines for animal welfare.

Cell culture. Naive OT-I $\mathrm{CD}^{+}{ }^{+}$or polyclonal $\mathrm{CD} 8{ }^{+}$cells were isolated using the positive $\mathrm{CD} 8{ }^{+}{ }^{-}$-cell isolation kit (Miltenyi Biotec) and polyclonal $\mathrm{CD} 4^{+}$cells were isolated using the positive $\mathrm{CD} 4^{+} \mathrm{T}$-cell isolation kit (Miltenyi Biotec), according to the manufacturer's protocols. For in-vitro T-cell activation, $\mathrm{T}$ cells were stimulated by $10 \mu \mathrm{g} \mathrm{ml}^{-1}$ plate-bound anti-CD3 and $10 \mu \mathrm{g} \mathrm{ml}^{-1}$ anti-CD28 Following activation for 2 days, $T$ cells were transferred into $75-\mathrm{cm}$ culture flask and maintained with fresh media supplemented with $10 \mathrm{ng} \mathrm{ml}^{-1} \mathrm{IL}-2$ (Peprotech) as needed. For imaging and transwell migration experiments, T cells were typically cultured in vitro for 4-6 days before use. 
Transwell migration assay. In vitro-activated polyclonal $\mathrm{CD} 8{ }^{+} \mathrm{T}$ cells were rested in the RPMI medium containing $1 \%$ fetal bovine serum at $37^{\circ} \mathrm{C}$ for $1 \mathrm{~h}$ before being loaded as a $100-\mu \mathrm{l}$ suspension of $10^{5}$ cells into the upper well (5-mm pore, Corning). hCCL ${ }^{* *}$ before or after ultraviolet deprotection, achieved by ultraviolet irradiation $\left(18 \mathrm{~mW} \mathrm{~cm}^{-2}\right)$ for $30 \mathrm{~s}$, was added to the bottom wells at indicated concentrations. Cells that transmigrated into the bottom well were enumerated $3 \mathrm{~h}$ later by flow cytometry with an internal cell counting standard.

Imaging of T-cell migration in vitro. DsRed-expressing $\mathrm{CD} 8^{+} \mathrm{T}$ cells were activated in vitro and rested in the RPMI medium containing $1 \%$ fetal bovine serum at $37^{\circ} \mathrm{C}$ on a $35-\mathrm{cm}$ dish (NEST) pre-coated with $7.5 \mu \mathrm{g} \mathrm{ml}{ }^{-1}$ recombinant mouse ICAM-2 (R\&D) in PBS for $1 \mathrm{~h}$ before imaging. To inhibit PI3K activities, the $\mathrm{T}$ cells were pre-treated with $100 \mathrm{nM}$ Wortmannin (Sigma) for $1 \mathrm{~h}$ before imaging and maintained in Wortmannin-containing media during imaging. Images were acquired using a Nikon A1RMP microscope equipped with Mai Tai DeepSee laser (Spectra-Physics), a 561-nm laser (Coherent) and $\times 60$ oil objective lens (Nicon). Acquisition was controlled by NIS-Elements software (Nikon). Data were processed by NIS-Elements Analysis software (Nikon) and Imaris (Bitplane).

Imaging CD8 T-cell interactions with target cells. For cell-cell interaction imaging, in vitro-activated $\mathrm{CD}^{+}$cells were incubated with $20 \mathrm{pg} \mathrm{ml}^{-1}$ $\mathrm{OVA}_{257-264}$ for $30 \mathrm{~min}$, then washed with $1 \times \mathrm{PBS}$ for five times. A total of $10^{5}$ $\mathrm{CD} 8{ }^{+} \mathrm{T}$ cells and $10^{5}$ peptide-pulsed $\mathrm{CD} 4{ }^{+}$cells were loaded onto the ICAM pre-coated $35-\mathrm{cm}$ dish and rested for $30 \mathrm{~min}$ before imaging.

Two-photon intravital imaging of T-cell chemotaxis. For in vivo imaging of T-cell migration in the dermal tissue, $\sim 10^{6}$ activated GFP-expressing CD8 ${ }^{+} \mathrm{T}$ cells were loaded into $20 \mu \mathrm{l}$ PBS containing $50 \mathrm{ng} \mathrm{\mu l}^{-1} \mathrm{hCCL}^{* *}$ and intradermally injected into the ear of albino B6 mice. Imagining was conducted at least $1 \mathrm{~h}$ after the injection and the field of view was chosen not to be directly at the injection site. For in vivo imaging of T-cell migration in the lymph node, the basic procedure was essentially as described previously ${ }^{23}$. Briefly, $3 \times 10^{6}$ activated GFP-expressing $\mathrm{CD}^{+} \mathrm{T}$ cells were intravenously transferred into B6 mice the day before and $20 \mu \mathrm{g}$ hCCL5 ${ }^{* *}$ was intraveneously injected $30 \mathrm{~min}$ before imaging. An infrared laser tuned to $920 \mathrm{~nm}$ (Mai Tai DeepSee, Spectra-Physics) was used to image GFPexpressing cells and a second laser tuned to $720 \mathrm{~nm}$ to photostimulate the region of interest. Photostimulation was always in a continuous mode in sync with imaging. Four-dimensional data sets were analysed using Imaris software (Bitplane). Cell migration was analysed by Imaris cell tracking module aided with manual correction and verification. Cell tracks that lasted for $<1$ min were excluded from analysis. For analysis of the mean chemotactic index within $10 \mathrm{~min}$ of photostimulation, cells with a path length of $<50 \mu \mathrm{m}$ were excluded, because they often leave the imaging volume too soon. AfterEffects and Illustrator were used to produce time-lapse image sequences and movies.

Statistical analysis. Unless indicated otherwise, $t$-tests were used to compare endpoint means of different groups. Statistic test and graphing were done with Prism (GraphPad).

\section{References}

1. Qi, H., Kastenmuller, W. \& Germain, R. N. Spatiotemporal basis of innate and adaptive immunity in secondary lymphoid tissue. Annu. Rev. Cell Dev. Biol. 30, 141-167 (2014).

2. Rot, A. \& von Andrian, U. H. Chemokines in innate and adaptive host defense: basic chemokinese grammar for immune cells. Annu. Rev. Immunol. 22, 891-928 (2004).

3. Swaney, K. F., Huang, C. H. \& Devreotes, P. N. Eukaryotic chemotaxis: a network of signaling pathways controls motility, directional sensing, and polarity. Annu. Rev. Biophys. 39, 265-289 (2010).

4. Insall, R. H. Understanding eukaryotic chemotaxis: a pseudopod-centred view. Nat. Rev. Mol. Cell Biol. 11, 453-458 (2010).

5. Kay, R. R., Langridge, P., Traynor, D. \& Hoeller, O. Changing directions in the study of chemotaxis. Nat. Rev. Mol. Cell Biol. 9, 455-463 (2008).

6. Allen, S. J., Crown, S. E. \& Handel, T. M. Chemokine: receptor structure, interactions, and antagonism. Annu. Rev. Immunol. 25, 787-820 (2007).

7. Bang, D. \& Kent, S. B. A one-pot total synthesis of crambin. Angew. Chem. Int. Ed. Engl. 43, 2534-2538 (2004).

8. Dawson, P. E., Muir, T. W., Clark-Lewis, I. \& Kent, S. B. Synthesis of proteins by native chemical ligation. Science 266, 776-779 (1994).

9. Fang, G. M. et al. Protein chemical synthesis by ligation of peptide hydrazides. Angew. Chem. Int. Ed. Engl. 50, 7645-7649 (2011).

10. Zheng, J. S., Tang, S., Qi, Y. K., Wang, Z. P. \& Liu, L. Chemical synthesis of proteins using peptide hydrazides as thioester surrogates. Nat. Protoc. 8, 2483-2495 (2013).
11. Rek, A., Brandner, B., Geretti, E. \& Kungl, A. J. A biophysical insight into the RANTES-glycosaminoglycan interaction. Biochim. Biophys. Acta 1794, 577-582 (2009).

12. Pakianathan, D. R., Kuta, E. G., Artis, D. R., Skelton, N. J. \& Hebert, C. A. Distinct but overlapping epitopes for the interaction of a CC-chemokine with CCR1, CCR3 and CCR5. Biochemistry 36, 9642-9648 (1997).

13. Baumann, L. \& Beck-Sickinger, A. G. Photoactivatable chemokines--controlling protein activity by light. Angew. Chem. Int. Ed. Engl. 52, 9550-9553 (2013).

14. Germain, R. N., Miller, M. J., Dustin, M. L. \& Nussenzweig, M. C. Dynamic imaging of the immune system: progress, pitfalls and promise. Nat. Rev. Immunol. 6, 497-507 (2006).

15. Momotake, A., Lindegger, N., Niggli, E., Barsotti, R. J. \& Ellis-Davies, G. C. The nitrodibenzofuran chromophore: a new caging group for ultra-efficient photolysis in living cells. Nat. Methods 3, 35-40 (2006).

16. Hirsch, E. et al. Central role for $G$ protein-coupled phosphoinositide 3-kinase gamma in inflammation. Science 287, 1049-1053 (2000).

17. Funamoto, S., Meili, R., Lee, S., Parry, L. \& Firtel, R. A. Spatial and temporal regulation of 3-phosphoinositides by PI 3-kinase and PTEN mediates chemotaxis. Cell 109, 611-623 (2002).

18. Hannigan, M. et al. Neutrophils lacking phosphoinositide 3-kinase gamma show loss of directionality during $\mathrm{N}$-formyl-Met-Leu-Phe-induced chemotaxis. Proc. Natl Acad. Sci. USA 99, 3603-3608 (2002).

19. Iijima, M. \& Devreotes, P. Tumor suppressor PTEN mediates sensing of chemoattractant gradients. Cell 109, 599-610 (2002).

20. Hoeller, O. \& Kay, R. R. Chemotaxis in the absence of PIP3 gradients. Curr. Biol. 17, 813-817 (2007).

21. Ferguson, G. J. et al. $\mathrm{PI}(3) \mathrm{K} \gamma$ has an important context-dependent role in neutrophil chemokinesis. Nat. Cell Biol. 9, 86-91 (2007).

22. Afonso, P. V. \& Parent, C. A. PI3K and chemotaxis: a priming issue? Sci. Signal. 4, pe22 (2011).

23. Xu, H. et al. Follicular T-helper cell recruitment governed by bystander B cells and ICOS-driven motility. Nature 496, 523-527 (2013).

24. Turner, L., Ward, S. G. \& Westwick, J. RANTES-activated human T lymphocytes. A role for phosphoinositide 3-kinase. J. Immunol. 155, 2437-2444 (1995).

25. Lammermann, T. et al. Neutrophil swarms require LTB4 and integrins at sites of cell death in vivo. Nature 498, 371-375 (2013).

26. Kent, S. B. H. Total chemical synthesis of proteins. Chem. Soc. Rev. 38, 338-351 (2009).

27. Zheng, J. S. et al. A new method for synthesis of peptide thioesters via irreversibleN-to-S acyl transfer. Org. Lett. 16, 4908-4911 (2014).

\section{Acknowledgements}

This work was funded in part by the Ministry of Science and Technology ' 973 ' programme (grant numbers 2014CB542501 and 2013CB932800), National Natural Science Foundation of China (grant numbers 81330070, 91313301, 81072464, 21225207 and 21402206), Tsinghua University Initiative Scientific Research Program (number 20131089224) and the Tsinghua-Peking Center for Life Sciences. H.Q. and L.L. were Tsinghua-Bayer Investigators and Tsinghua-Janssen Investigators.

\section{Author contributions}

X.C. designed and conducted biological experiments including in-vitro and in-vivo imaging work, with assistance from R.Z., W.S. and H.Z. S.T. and J.-S.Z. carried out most of synthetic work with assistance from Z.-P.W., H.-N.C. and J.-Y.C. H.Q. and L.L. conceptualized the study, supervised the research and wrote the manuscript with assistance from S.T. and X.C. All authors contributed collectively to data analysis and interpretation.

\section{Additional Information}

Supplementary Information accompanies this paper at http://www.nature.com/ naturecommunications

Competing financial interests: The authors declare no competing financial interests.

Reprints and permission information is available online at http://npg.nature.com/ reprintsandpermissions/

How to cite this article: Chen, X. et al. Chemical synthesis of a two-photon-activatable chemokine and photon-guided lymphocyte migration in vivo. Nat. Commun. 6:7220 doi: $10.1038 /$ ncomms8220 (2015).

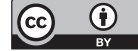

This work is licensed under a Creative Commons Attribution 4.0 International License. The images or other third party material in this article are included in the article's Creative Commons license, unless indicated otherwise in the credit line; if the material is not included under the Creative Commons license, users will need to obtain permission from the license holder to reproduce the material. To view a copy of this license, visit http://creativecommons.org/licenses/by/4.0/ 